\title{
A unified account of inquisitive and assertive rising declaratives
}

\author{
Daniel Goodhue*
}

\begin{abstract}
Previous work on rising declaratives has argued that some have an inquisitive interpretation similar to polar questions, and that this meaning is intonationally distinguished by a steep final rise to a high boundary tone, while others have an assertive interpretation, similar to assertions of falling declaratives, that has a shallower final rise to a lower, high boundary tone. I demonstrate that this strict form-meaning correlation does not hold because there are inquisitive rising declaratives that have a shallow final rise. I argue for a unified theory of rising declaratives with enough interpretational flexibility to explain these crosscutting patterns.
\end{abstract}

Keywords. rising declaratives; biased questions; speech acts; pragmatics; semantics; intonation; prosody

1. Introduction. Rising declaratives provide a useful test case for theories of the semanticspragmatics interface that aim to explain why the main clause types, when they are the root clause, are canonically linked to certain discourse functions. For example, matrix declaratives are canonically used to assert, and thus usually commit the speaker to their propositional content and signal the goal of updating the common ground with it. Some argue that declaratives are designated for assertions by an illocutionary force operator or an extra-grammatical convention of use (e.g. Gunlogson 2003; Lauer 2013; a.o.), while others propose that their assertoric function is derivable from their semantic denotation combined with a particular view of dynamic pragmatics (e.g. Farkas \& Roelofsen 2017; Portner 2018; a.o.).

However, produce a root declarative with the rising intonation typically used in a polar question in English, and it now seems to be used to ask a question, albeit one with a special pragmatic requirement that there be some contextual bias in favor of the proposition denoted by the clause. Any attempt to explain the canonical discourse functions of matrix clauses will need to explain this exception to the apparent rule for matrix declaratives.

In this paper, I argue that the empirical situation is more complicated than I have described so far, and that dealing with this complication speaks in favor of a particular view of the semantics-pragmatics interface. The complication is that, while rising declaratives are frequently used to ask questions, they can also be used to make assertions much like falling declaratives, and there is reason to believe that these disparate uses deserve a unified account. Articulating a unified account reveals that much of the illocutionary force of utterances must be pragmatically derived. In particular, intonation cannot directly signal whether or not the speaker intends to commit to the truth of the content of the matrix declarative clause (pace Gunlogson 2003, Truckenbrodt 2012, Farkas \& Roelofsen 2017, Jeong 2018, Rudin 2018, among others). The audience can only work out whether the speaker intends to commit based on the semantic content, the intonational contribution, and the context of utterance.

\footnotetext{
* Thank you to Valentine Hacquard, Sunwoo Jeong, Jeffrey Lidz, Deniz Rudin, Bernhard Schwarz, Michael Wagner, Alexander Williams, and Yu'an Yang for helpful discussion. Thank you also to the audiences at the LSA Annual Meeting, the ZAS workshop on Biased Questions: Experimental Results \& Theoretical Modelling, the Rutgers Linguistics Colloquium, and to two seminars and the General Linguistics Lab Meeting (GLLaM) at the University of Maryland. All mistakes are my own. Author: Daniel Goodhue, University of Maryland (dgoodhue@umd.edu).
} 
In section 2, I establish the empirical phenomenon of rising declaratives. In section 3, I introduce novel data to demonstrate that some rising declaratives used to ask questions cannot be distinguished intonationally from those used to assert. This leads me to argue that the two uses of rising declaratives deserve a unified account. I show how this is a challenge to prior work, and then build on that work to offer a unified account in section 4 .

\section{Background on rising declaratives.}

2.1. THE DIFFERENCE BETWEEN INQUISITIVE RISING DECLARATIVES AND POLAR INTERROGATIVES. A rising declarative is an utterance of a matrix declarative clause produced with a rising intonation that, to a first approximation, is similar to the intonation produced in polar questions in English, typically assigned the ToBI annotation L* H-H\% (Pierrehumbert 1980), represented here via ' $\nearrow$ '. Consider the classic example of a rising declarative in (1-a).

(1) $\mathrm{S}$ is in her office. A has just arrived holding a wet umbrella and raincoat.

a. S: Hey! It's raining $\nearrow$

b. S: Hey! Is it raining $\nearrow$ (based on Gunlogson 2003 p. 96)

(1) is a context in which both the rising declarative and the polar interrogative are felicitous means of asking a question about whether it's raining.

Now consider (2).

(2) $\mathrm{S}$ is in her office. A has just arrived, and exhibits no evidence whatsoever about the weather outside.

a. S: \# Hey! It's raining $\nearrow$

b. S: Hey! Is it raining $\nearrow$

(based on Gunlogson 2003 p. 95)

Here, only the polar interrogative is felicitous. This suggests that the felicity of the rising declarative depends on the contextual evidence in favor of the proposition that it is raining in the form of A's wet umbrella and raincoat.

Finally, A can reply by asserting that it's raining, using a falling declarative.

A: (Yeah) It's raining \

These patterns raise the following questions: What are the roles of clause type and intonation in producing questions and assertions? How do we explain the unique contextual bias of rising declaratives? These questions have animated much work in recent years, in part because accounting for this data provides the opportunity to discover something about the boundary between semantics and pragmatics, and the roles of each in producing the discourse effects of utterances (see e.g. Gunlogson 2003, 2008; Truckenbrodt 2006, 2012; Malamud \& Stephenson 2015; Farkas \& Roelofsen 2017; Krifka 2017; Westera 2017, 2018; Jeong 2018; Rudin 2018).

2.2. Assertive Rising DeClaratives. Complicating this effort to chart the semanticspragmatics interface is the fact that not all rising declaratives are used to ask questions about the content of the declarative clause. That is, while (1-a) is an inquisitive rising declarative, there are also assertive rising declaratives, as in (4) and (5).

(4) A: Do you speak Spanish?

S: I speak Ladino $\nearrow$

(Jeong 2018 p. 307, Farkas \& Roelofsen 2017 p. 239, Ward \& Hirschberg 1985 p. 765) 
A: What are you eating?

$\mathrm{S}$ : This is a persimmon $\nearrow$

While S's utterances in (4) and (5) are clearly assertions, and thus commit the speaker to the propositional content of the declarative, they nevertheless convey some kind of secondary question. In (5), this is roughly, "Have you heard of persimmons? Is the name enough information?". (4) could convey a similar kind of question, but more likely it conveys something like, "Is Ladino close enough to Spanish? Does it count for your purposes?".

Prior accounts of rising declaratives set the assertive variety aside as something distinct. Farkas \& Roelofsen write:

"We assume for now that [assertive rising declaratives] are of a different nature than the ones we are concerned with, although ultimately we would of course like to better understand the connection between the different varieties and perhaps even come to a fully unified theory."

(Farkas \& Roelofsen 2017 p. 239)

Jeong (2018), building on Hirschberg \& Ward 1995, argues that there is a principled reason to treat inquisitive and assertive rising declaratives as distinct, namely she argues that the two meanings are associated with different intonational contours. In particular, inquisitive rising declaratives are claimed to rise more steeply $\left(\mathrm{L}^{*} \mathrm{H}-\mathrm{H} \%\right)$, while the assertive variety have a shallower rise $\left(\mathrm{H}^{*} \mathrm{H}-\mathrm{H} \%\right)$. If this is correct, then each rise could make a different meaning contribution, resulting in the inquisitive/assertive interpretational split observed above.

2.3. Filling OUT THE TAXONOMY OF RISING DECLARATIVES. In section 3, I will argue that the intonational distinction discussed by Hirschberg \& Ward (1995) and Jeong 2018 does not track the distinction between inquisitive and assertive rising declaratives. In preparation for that argument, I need to make the taxonomy of rising declaratives slightly more precise. ${ }^{2}$

\footnotetext{
${ }^{1}$ Note that while S's utterances in (4) and (5) are both appropriate with the rising intonation of rising declaratives, (4) would also be appropriate, and perhaps even preferred, with the rise-fall-rise contour (RFR, transcribed as $\mathrm{L}^{*}+\mathrm{H}$ L-H\%, see e.g. Goodhue et al. 2016). In fact, (4) is based on an example that Ward \& Hirschberg (1985) originally introduced to exemplify RFR, not assertive rising declaratives. Importantly, (5) (and also (13) and (16) below) is not felicitous with RFR, but is perfectly felicitous with the rise typical of rising declaratives. This shows that assertive rising declaratives and declaratives with RFR are not in free variation with one another. If they were, then given that (4) may even be preferred with RFR, it would be reasonable to wonder whether assertive rising declaratives are a coherent category of their own, or if they might just be a slightly non-standard production of what is meant to be an asserted declarative with RFR contour. The fact that they are not in free variation lends support to the view I will ultimately take, that assertive rising declaratives must be accounted for qua rising declaratives.

2 Jeong's (2018) taxonomy of rising declaratives is more complex than (6), identifying "contradictory RDs" as a third kind of inquisitive RD, and subdividing assertive RDs into "unsure about a metalinguistic issue" and "uptalk". I think contradictory RDs are a special kind of incredulous RD, distinguished by whether the speaker intends to ultimately accept the propositional content of the RD or not, which is due to how the speaker views their own knowledge relative to the addressee's. The label "uptalk" is sometimes used to refer to the continuation rises, but I take the relevant phenomenon of interest to be a certain turn-final utterance demonstrated by Jeong (p. 307). I suspect uptalk may have a genuinely distinct intonation, and leave it to future work.
} 


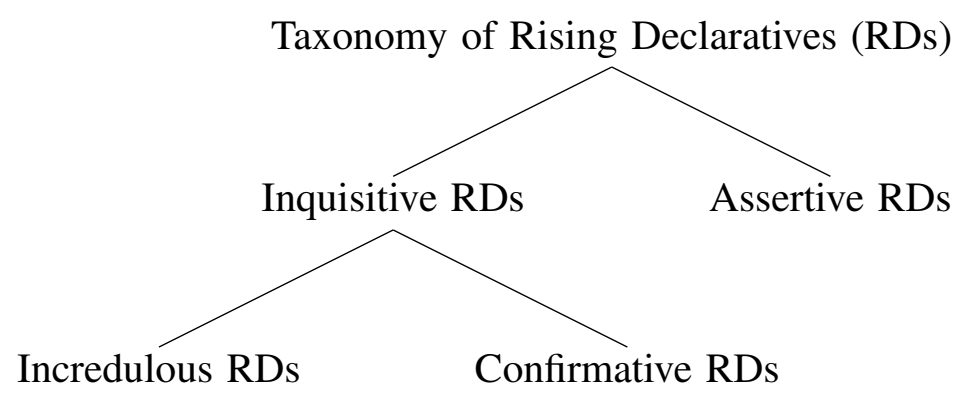

The inquisitive RD in (1-a) could have been incredulous or confirmative, depending on how surprised the speaker was by the evidence of rain. (7) and (8) distinguish these two readings.

\section{(7) Incredulous}

S knows John well, and believes that he doesn't have any siblings.

A: John went to the airport to pick up his sister.

S: John has a sister $\nearrow \quad$ (based on Jeong 2018 p. 307, based on Gunlogson 2003 p. 132)

Intuitively, the rising declarative in (7) is used to express that $S$ is incredulous of the content of the declarative clause, which was presupposed by A's utterance. S may ultimately go on to accept that proposition as true, or to maintain that it is false, depending on how confident $\mathrm{S}$ is in their prior belief that John has no siblings.

\section{(8) Confirmative}

$\mathrm{S}$ and A made plans two days ago to get drinks tonight. They haven't spoken about it since.

S: We're still on for tonight $\nearrow$

(Rudin 2018 p. 37)

The rising declarative in (8) does not express that $\mathrm{S}$ did not previously believe the content of the declarative. On the contrary, S seems to believe that that proposition is highly likely to be true, and is using the rising declarative to double check its truth with A.

Despite the differences between them, both incredulous and confirmative RDs share the following properties in common that distinguish them as inquisitive.

(9) Meaning generalizations for Inquisitive RDs

a. the speaker makes no new commitments

b. the speaker expects the addressee to answer

c. the speaker anticipates that the addressee will commit to the proposition denoted by the declarative clause

Inquisitive rising declaratives share the properties in (9-a) and (9-b) with matrix uses of polar interrogatives like (1-b). The property in (9-c) is the contextual bias condition that distinguishes inquisitive rising declaratives from standard polar questions.

(9) can be contrasted against the meaning properties of assertive rising declaratives like (4) and (5), collected in (10).

(10) Meaning generalizations for Assertive RDs

a. the speaker commits to the proposition denoted by the declarative clause

b. the speaker does not necessarily expect the addressee to be committed to it

c. the speaker does not expect the addressee to answer the rising declarative as if it 
were a question about whether its propositional content is true

d. the utterance of the rising declarative raises a second issue

Assertive rising declaratives share the properties in (10-a) through (10-c) with matrix uses of falling declaratives like (3). The property in (10-d) distinguishes assertive rising declaratives from standard assertions of falling declaratives.

3. The intonational distinction does not track the inquisitive/assertive split. In section 3.1 , I introduce two minimal triples to enable comparison of the intonation used in each of the three kinds of rising declaratives in (6), namely the two sub-kinds of inquisitive rising declaratives, incredulous and confirmative, and assertive rising declaratives. The result, discussed in section 3.2, will be that confirmative and assertive rising declaratives pattern together intonationally to the exclusion of incredulous rising declaratives, and thus intonation does not track the inquisitive/assertive interpretational split.

3.1. IntONAtional ANALYSis of TWO Minimal tRiples. Consider the first minimal triple.

(11) Incredulous (IRD)

$\mathrm{S}$ and $\mathrm{A}$ are watching a girl give a very professional performance in a school debate. From this, $\mathrm{S}$ is thinking that she might be 12 or 13 years old.

S: She's amazing.

A: I know, and she's only 9 years old.

S: She's nine $\nearrow$

(12) Confirmative (IRD)

$\mathrm{S}$ and A are on their way to a birthday party for the daughter of A's friend. They stop at a store to get a birthday card. As they are both scanning the display for a card for the correct age, $S$ is trying to remember how old the girl has just turned, and he thinks he remembers A telling him that she just turned nine, but he wants to confirm it.

S: She's nine $\nearrow$

(13) Assertive (ARD)

$\mathrm{S}$ is enrolling his daughter in a summer camp program with the camp organizer $\mathrm{A}$.

S: I want to sign her up for Spanish classes in the mornings, and rock climbing in the afternoons.

A: Okay, there are limited places in each activity based on age group, and some of the age groups have already filled up for rock climbing. How old is your daughter?

S: She's nine $\nearrow$

In terms of meaning, the incredulous and confirmative RDs pattern together to the exclusion of the assertive RD in that (11) and (12) exhibit the properties in (9) while (13) instead exhibits the properties in (10) (the second issue raised in (13), to be discussed further below, is roughly "Is there still room in the 9-year-old's rock climbing group?").

And yet, the confirmative RD in (12) is naturally produced with a shallower rise, making it intonationally similar to the assertive RD in (13). Pitch tracks for these examples, given in Figure 1, reveal that the most obvious distinction here is in the height of the final boundary tone: the confirmative and assertive RDs terminate in a similar high boundary tone a little over $200 \mathrm{~Hz}$, while the incredulous RD rises to a much higher boundary tone.

The intuition is clear: Given a minimal triple that distinguishes the three readings, confir- 


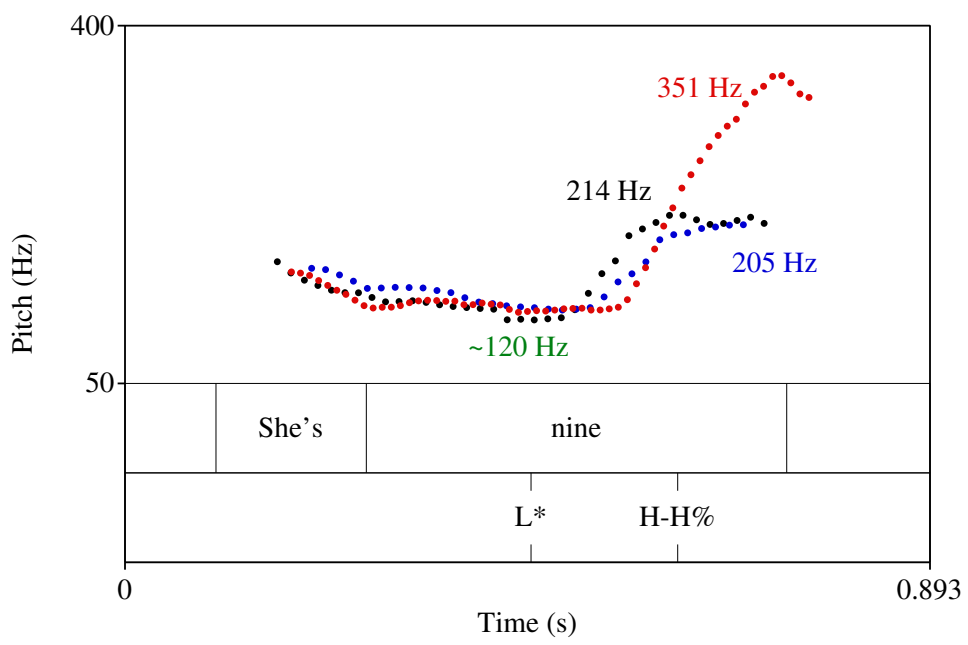

Figure 1. Pitch tracks for the incredulous (11) in red, the confirmative (12) in blue, and the assertive (13) in black, with the max $\mathrm{Hz}$ of final boundary tones listed, and the average $\mathrm{Hz}$ for the $\mathrm{L}^{*}$ pitch accent in green. All pitch tracks given in this paper are produced from recordings of the author, a native speaker of North American English, using Praat (Boersma \& Weenink 2020).

mative RDs pattern intonationally with assertive RDs by having a lower boundary tone, to the exclusion of incredulous RDs. The pattern will be replicated with (14)-(16) below.

Beyond the boundary tone, all three contours have a similar shape, and a similar low pitch accent on nine. Table 1 gives the precise pitch minima and maxima for each of these productions. Note that the assertive RD in (13) has a pitch accent that is 1 to 1.5 semitones lower than the other two. This distinction is actually the opposite of what would be expected based on the prior literature, to be discussed in section 3.2. However, the height of the pitch accents pattern differently in the following minimal triple, (14)-(16).

\begin{tabular}{lrr} 
& Nuclear pitch accent & Final boundary tone \\
\hline Incredulous RD in (11) & $122 \mathrm{~Hz}$ & $351 \mathrm{~Hz}$ \\
Confirmative RD in (12) & $125 \mathrm{~Hz}$ & $205 \mathrm{~Hz}$ \\
Assertive RD in (13) & $115 \mathrm{~Hz}$ & $214 \mathrm{~Hz}$ \\
\hline
\end{tabular}

Table 1. Pitch minima and maxima for each of the productions in fig. 1.

\section{(14) Incredulous (IRD)}

Suppose that a rent in the amount of $\$ 999$ is surprising to $\mathrm{S}$ (either because it's high or low in S's market, whichever is easier to imagine).

$\mathrm{S}$ : How much is the rent?

A: Nine ninety-nine.

S: It's nine ninety-nine $\nearrow$

(15) Confirmative (IRD)

Suppose $\mathrm{S}$ is writing a rent check for his college student child A. This is only the second time S has had to pay for A's rent, and he is looking for confirmation that it's $\$ 999$. S, pen in hand, hovering over his checkbook, vaguely remembers that A's rent is $\$ 999$, 
but wants to double check.

S: It's nine ninety-nine $\nearrow$

Assertive (ARD)

A works for a bank, and $\mathrm{S}$ has called to try to figure out whether he can pay his rent electronically. $\mathrm{S}$ is certain that the rent is $\$ 999$ (it's his own rent), but A given what A says below, $\mathrm{S}$ can infer that Zelle pay must have an upper limit on payment amounts, thus $S$ is uncertain about whether Zelle pay will allow a payment of $\$ 999$.

A: We have Zelle pay, it's like PayPal. How much is your rent?

S: It's nine ninety-nine $\nearrow$

Again, the incredulous and confirmative RDs pattern together to the exclusion of the assertive RD in terms of meaning, while the confirmative and assertive RDs pattern together to the exclusion of the incredulous RD in terms of intonation. Figure 2 shows that the intonational pattern of the nuclear contours (from the final pitch accent on) mimics those in fig. 1: the confirmative and assertive RDs terminate in a lower high boundary tone than the incredulous RD.

Moreover, the fact that this triple has longer target sentences means that there is a prenuclear pitch accent on the first nine. Interestingly, this interacts with the contour in the incredulous RD, resulting in an initial rise. This same effect has been demonstrated experimentally by Goodhue et al. (2016). I believe that this is a pre-nuclear intermediate phrase that is identical phonologically to the nuclear contour, i.e. L* $\mathrm{H}-$. It then falls again to reach the $\mathrm{L}^{*}$ nuclear pitch accent. I will discuss the possible implications of this in section 3.2.

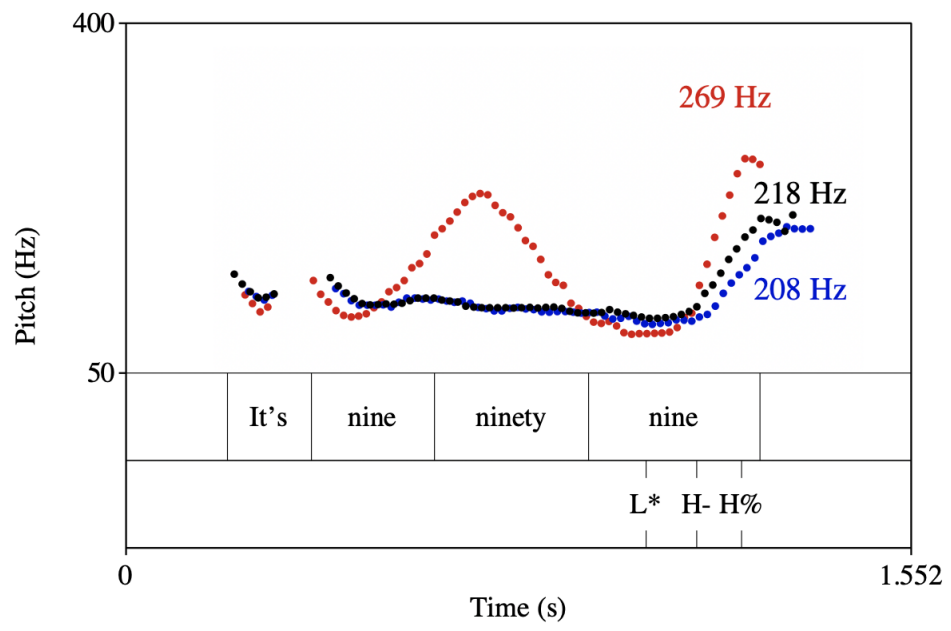

Figure 2. Pitch tracks for the incredulous (14) in red, the confirmative (15) in blue, and the assertive (16) in black, with the max $\mathrm{Hz}$ of final boundary tones listed.

The pitch minima and maxima for (14)-(16) (all located in the nuclear contour) can be found in table 2. This time the incredulous RD has a nuclear pitch accent that is 1.3 to 2 semitones lower than the other two. This variation across the two minimal triples suggests that there might not be any systematic difference in the height of the pitch accents across the three kinds of RDs. A multi-participant production experiment would be needed to settle this.

3.2. Summary AND implications. The upshot of these results are summarized in table 3. What I have shown is that there is an intuitive intonational distinction across sub-kinds of ris- 


\begin{tabular}{lrr} 
& Nuclear pitch accent & Final boundary tone \\
\hline Incredulous RD in (11) & $112 \mathrm{~Hz}$ & $269 \mathrm{~Hz}$ \\
Confirmative RD in (12) & $121 \mathrm{~Hz}$ & $208 \mathrm{~Hz}$ \\
Assertive RD in (13) & $126 \mathrm{~Hz}$ & $218 \mathrm{~Hz}$ \\
\hline
\end{tabular}

Table 2. Pitch minima and maxima for each of the productions in fig. 2.

ing declaratives. Some RDs rise more steeply to a higher final boundary tone than others, but that distinction does not track the distinction in meaning between inquisitive and assertive rising declaratives. Instead, steeper rises to higher final boundary tones correlate positively with incredulousness. Meanwhile, confirmative and assertive rising declaratives pattern together intonationally, despite that the former is inquisitive while the latter is assertive.

\begin{tabular}{l||c|c|c}
\hline \multicolumn{1}{l||}{ Kinds of RDs } & \multicolumn{2}{c}{ Inquisitive } & Assertive \\
\hline Sub-kinds & Incredulous & Confirmative & Assertive \\
\hline Intonation & Steep rise to higher $\mathrm{H} \%$ & \multicolumn{2}{c}{ Shallow rise to lower $\mathrm{H} \%$} \\
\hline
\end{tabular}

Table 3. The inquisitive/assertive meaning distinction in rising declaratives does not correlate with an intonational distinction.

This is unexpected based on prior work on both intonational phonology and intonational meaning. In summary, various researchers have claimed that assertive rising declaratives have a unique contour of their own, and develop semantic/pragmatic accounts that depend on them having a unique intonation (e.g., Hirschberg \& Ward 1995, Truckenbrodt 2012, Westera 2017, 2018, Jeong 2018, and Rudin 2018). Thus they predict that productions of assertive RDs should stand apart intonationally from inquisitive RDs, which is not what we find. This calls into question the view that assertive rising declaratives can be circumscribed as distinct from inquisitive rising declaratives, and have their unique interpretation pinned on a distinct contribution from a unique intonational contour. It speaks in favor of giving a unified account of inquisitive and assertive rising declaratives.

These empirical facts pose a challenge for prior work on intonational phonology: Pierrehumbert (1980) and Pierrehumbert \& Hirschberg (1990) distinguish two related nuclear contours, $\mathrm{L}^{*} \mathrm{H}-\mathrm{H} \%$ and $\mathrm{H}^{*} \mathrm{H}-\mathrm{H} \%$. L* $\mathrm{H}-\mathrm{H} \%$ is the standard nuclear contour for English polar questions and also inquisitive rising declaratives, and various researchers have claimed that $\mathrm{H}^{*}$ $\mathrm{H}-\mathrm{H} \%$ is reserved for assertive rising declaratives (e.g., Hirschberg \& Ward 1995, and Truckenbrodt 2012). Therefore, this view predicts that assertive and inquisitive RDs should differ in the height of the nuclear pitch accent, with inquisitive RDs having lower pitch accents. ${ }^{3}$ The contours in figures 1 and 2 challenge this prediction, as the systematic distinction is in

\footnotetext{
${ }^{3}$ Technically, this conclusion does not immediately follow. Pierrehumbert (1980) writes that $\mathrm{H}^{*} \mathrm{H}-\mathrm{H} \%$ is like L* H-H\% "except that the nuclear accent is on the same level as the phrase accent." (p. 63) The most intuitive way to understand this statement is as claiming that the $\mathrm{H}^{*}$ in $\mathrm{H}^{*} \mathrm{H}-\mathrm{H} \%$ is higher in a given speaker's range than the $\mathrm{L}^{*}$ in $\mathrm{L}^{*} \mathrm{H}-\mathrm{H} \%$. However the statement would also be true if $\mathrm{H}^{*}$ and $\mathrm{L}^{*}$ were each at the same height, and $\mathrm{H}$ - were lower in $\mathrm{H}^{*} \mathrm{H}-\mathrm{H} \%$ than in $\mathrm{L}^{*} \mathrm{H}-\mathrm{H} \%$. The latter is counterintuitive given the use of ' $\mathrm{H}(\mathrm{igh})$ ' and ' $\mathrm{L}(\mathrm{ow})$ ', but it better predicts the phonetic reality that I observed in figures 1 and 2. Figure 14.13 in Pierrehumbert \& Hirschberg 1990 (p. 281) as well Hirschberg \& Ward's (1995, p. 409) discussion suggests that the $H^{*}$ is intended to appear higher than the $\mathrm{L}^{*}$ in the speaker's range across these two nuclear contours. Thus I assume that the intuitive understanding of the ToBI notation was intended by the authors of prior work for these two contours.
} 
the height of the boundary tones, not the pitch accents.

The empirical facts also pose a challenge to Hirschberg \& Ward (1995) and Truckenbrodt (2012), who both propose meanings for individual tones in these precise ToBI annotations. For example, Truckenbrodt analyzes $\mathrm{H}^{*}$ as conveying that the proposition denoted by the declarative clause is being added to the common ground, and this is so whether it is present in the rising $\mathrm{H}^{*} \mathrm{H}-\mathrm{H} \%$, or the falling $\mathrm{H}^{*} \mathrm{~L}-\mathrm{L} \%$, standardly used in assertions of falling declaratives. Meanwhile, $\mathrm{H}$ - is analyzed as conveying that a proposition is questioned. In $\mathrm{H}^{*} \mathrm{H}-\mathrm{H} \%$ contours, this is the secondary issue raised by an assertive rising declarative, while in $\mathrm{L}^{*} \mathrm{H}-\mathrm{H} \%$ contours, it is the propositional content of the utterance itself. Finally, L* and L- are treated as a meaningless defaults. Thus, if assertive RDs alone bore $\mathrm{H}^{*} \mathrm{H}-\mathrm{H} \%$ contours, then this analysis would quite elegantly predict the generalizations in (9) and (10). But the data above show that assertive RDs do not obviously have a higher pitch accent than inquisitive RDs.

The empirical facts above also challenge the account in Jeong 2018, and Rudin 2018 who shares Jeong's intonational assumptions, since they predict inquisitive and assertive RDs to have different intonations that make distinct meaning contributions resulting in the interpretational asymmetry. Jeong correctly notes that contextual pragmatics might be able to override the interpretational contribution of a contour, leading listeners to assume that they have heard one or the other rise based on whichever interpretation is most appropriate in that context. Even so, the claim that there are two phonologically distinct contours with distinct meanings predicts speakers to produce those distinct contours given appropriately disambiguated contexts. But this is not the case for confirmative and assertive RDs in the minimal triples above.

Finally, my view of the intonational facts can explain the interesting experimental results in Jeong 2018 (experiments 1 \& 2, p. 315ff.). Jeong ran perception studies in which participants listened to utterances and judged whether they were assertions or questions. She produced three distinct rising declaratives by manipulating the height of the boundary tone, while holding the height of the pitch accent constant. She found that steepness correlated with inquisitive interpretations: the steepest rise elicited $65 \%$ question interpretations, the middle rise $62 \%$, and the shallowest rise 50\%. Here's how my view can explain these results: The steeper the rise, the more likely the speaker is conveying incredulity about the propositional content of the declarative. This is only consistent with an inquisitive interpretation, namely one in which the speaker does not make a commitment to the declarative content and expects an answer. So participants infer that the steepest rise marks an inquisitive RD. Shallower rises are consistent with both an inquisitive confirmative interpretation and an assertive interpretation, and thus participants were split at roughly 50\% in their interpretations for the shallowest rise.

4. A unified account. In section 3, I demonstrated that there is no intonational distinction that tracks the interpretational distinction between inquisitive and assertive rising declaratives, and that this poses challenges to certain views of intonational phonology and meaning. Given this, I think a unified account of inquisitive and assertive rising declaratives is called for. If a unified account can be given, it has the added the benefit of being more parsimonious. In a nutshell, I propose that there is only one relevant rising intonation in rising declaratives and that it contributes a not-at-issue lack of speaker commitment. Usually this lack of commitment is about the propositional content of the utterance, but sometimes it is about some other relevant issue. In order to allow the rise to contribute such a degree of flexibility, we need a very spare dynamic pragmatics: the only convention of use for declaratives and interrogatives is that their 
semantic content is added to the table. Further effects, such as speaker commitment, are part of the pragmatics.

To see why we need this pragmatic flexibility, consider two recent accounts that my account will build on, but that cannot account for assertive rising declaratives because the conventional discourse effects they assign to certain clause-intonation pairs are too rigid. Farkas $\&$ Roelofsen (2017) analyze rising intonation as a semantic operator that produces a polar question interpretation from a declarative clause. Combined with their proposed utterance function, the speaker only commits to the truth of the tautology that one of the answers $p$ or not- $p$ in the polar question denotation is true. Rudin (2018), based on an idea in Truckenbrodt 2006, instead treats intonation as a device for manipulating speaker commitment in the dynamic pragmatics: falling intonation commits the speaker to the propositional content of the utterance, while rising intonation does not. While these accounts differ in substantial ways, they both predict that illocutionary force is determined by clause type and intonation. In particular, rising intonation immediately implies that the speaker is not committed to the content of the declarative, and so is not asserting. In order to bring assertive rising declaratives into the fold, we need to weaken the effects of clause type and intonation at the semantics-pragmatics interface, so that the combination of the two does not immediately determine illocutionary force.

4.1. INGREDIENTS OF THE ACCOUNT. I assume that all declaratives have the same semantic denotation regardless of intonation, and likewise for interrogatives:

Hamblin (1973) semantics

a. Declarative: $\llbracket \phi \rrbracket=\{p\}$

b. Polar interrogative: $\llbracket ? \phi \rrbracket=\{p, \neg p\}$

I assume an abridged Farkas \& Bruce 2010 style model of context, which builds in turn on Stalnaker 1978, Roberts 1996/2012, and Gunlogson 2003, among others.

A context $c$ is a tuple $\langle C G, T, Q U D\rangle$

a. $C G$ is the common ground, the set of all propositions that all discourse participants are publicly committed to in $c$

b. $\quad T$ is a table, i.e. a push-down stack of issues (an issue is a set of propositions)

c. $Q U D$ is a contextually salient question/goal

I follow Rudin 2018 in assuming a $Q U D$ that is separate from $T$. The issue at the top if $T$ is the one that has most recently been raised. The $Q U D$ is an overarching question or goal of a conversation that can be distinct from the topmost issue of $T$. This distinction will become relevant in the treatment of assertive rising declaratives below.

I take intonation to contribute non-at-issue meaning, in line with prior work (e.g., Ward \& Hirschberg 1985, Goodhue \& Wagner 2018, among others). I propose that the rise associated with rising declaratives and polar questions ' $\nearrow$ ' signals the speaker's lack of commitment to the truth of a proposition $q$. By default, $q$ is identified with the content $p$ of the clause uttered. For declaratives, $p$ is the proposition in the singleton set denoted by the declarative in (17-a); for polar interrogatives, $p$ is the content proposition before the $\mathrm{Q}$ morpheme uses it to produce the doubleton set in (17-b).

In some contexts, it is implausible to assume this default because the speaker is clearly in a position to know whether $p$ is true, and to know more about $p$ than the addressee (cf. Hud- 
son 1975). In such cases, $q$ is not likely to be $p$, but is instead some proposition such that $q \cap p$ addresses the QUD, while $p$ alone would not. In other words, when it's implausible for the speaker to express lack of commitment to $p$, our effort to identify the $q$ that they lack commitment to is guided by relevance to the QUD. A formal implementation is given in (19). ${ }^{4}$

a. $\llbracket \nearrow \rrbracket^{c}=\lambda C_{\{\langle s, t\rangle\}}:|C|=1 . C$

b. Non-at-issue meaning:

There is a proposition $q$ s.t. the speaker is not committed to $q$

(i) $\quad \&$ there is a set of propositions $P$ s.t. $q \in P \& C \subseteq P \& \cap P$ is an answer to the QUD in $c \& \bigcap(P-\{q\})$ is not an answer to the QUD in $c$.

c. Default that can be violated: $q \in C$

The singleton set requirement of (19-a) forces ' $\nearrow$ ' to merge lower than the $\mathrm{Q}$ morpheme in polar interrogatives. This technical move enables the denotation to work with both declaratives and polar interrogatives. In particular, $C$ cannot be $\{p, \neg p\}$, otherwise $\bigcap P$ would be empty.

(19-b) defines the non-at-issue lack of commitment contribution. (19-b-i) ensures that the proposition $q$ that the speaker lacks commitment to is relevant to the QUD. Normal Gricean relevance requires the content of the matrix clause uttered, $C$, to be relevant to the QUD (Roberts 1996/2012). In a case where the default in (19-c) is not met, i.e. $q \notin C$, there could be many irrelevant propositions $q$ that the speaker is not committed to. Thus without the requirements in (19-b-i), it would be very easy to satisfy (19-b), and the account would overgenerate assertive rising declaratives. In the end, it may be preferable to say that Gricean relevance does not just require $C$ to be relevant to the QUD, but also the proposition $q$ that the rise expresses lack of commitment about. If so, then we could safely remove (19-b-i) and allow the pragmatics to exclude irrelevant expressions of lack of commitment. For the moment, I leave it in because it will help us to see how $q$ is identified in assertive rising declaratives.

I leave falling intonation ' $\searrow$ ' as an unmarked default. In using it, the speaker fails to explicitly mark lack of commitment, from which inferences can be drawn.

As for dynamic pragmatics, I assume that utterances of any declarative or interrogative $\phi$ has the effect of putting $\llbracket \phi \rrbracket$ on the table.

UTTERANCE $\left(\phi, c_{n}\right) \rightarrow c_{n+1}$ such that

a. $\quad T_{n+1}=T_{n}+\llbracket \phi \rrbracket$

Crucially, this utterance function lacks any notion of commitment, since inquisitive RDs do not commit the speaker to their content, while assertive RDs do. The speaker, in using a rising declarative, can intend to commit to the content of the declarative or not. The choice of declarative plus rise does not immediately settle whether the speaker makes a commitment. From the hearer's perspective, speaker commitment must be determined pragmatically.

On the view of pragmatics adopted here, conversation is about building common ground to shrink the context set and find out what the world is like (Stalnaker 1978; Roberts 1996/2012). Common ground is built by adding issues to the table, and removing them by agreeing to commit to answers to them (Farkas \& Bruce 2010, Rudin 2018). Commitment to a proposition $p$ requires the interlocutor to believe $p$ and to have evidence for $p$ (Gricean quality). Given pres-

\footnotetext{
${ }^{4}$ The functional type of the variable ' $C$ ' in (19-a) is $\langle\langle s, t\rangle, t\rangle$. I use ' $\{\langle s, t\rangle\}$ ' (the type of sets characterized by $\langle\langle s, t\rangle, t\rangle$ functions) because the denotation in (19) treats $C$ as a set of propositions in various places.
} 
sure to grow the common ground, and the means by which it is done, utterances of declaratives and interrogatives are regulated by the following pragmatic requirement.

Pragmatic requirement of support for a proposition $p$ in $I$

When an issue $I$ is added to the table $T$, there is pressure for an interlocutor to add a proposition $p \in I$ to their discourse commitments.

While others have discussed the pressure to remove issues from the table as a driving force of conversation, what's new here is that I want to use this drive as a key component in deriving the basic effects of asserting and questioning, in particular speaker commitment in assertions and the expectation that addressees answer questions.

4.2. Application to Key EXAmples. Consider the following four utterances by the speaker $\mathrm{S}$ and their contexts. (23) and (25) appeared in section 3 as (13) and (12) respectively, and are paired here with a falling declarative (22) and a polar interrogative (24).

\section{(22) Falling declarative}

A: How old is your daughter?

S: She's nine \}

(23) Assertive rising declarative

$\mathrm{S}$ is enrolling his daughter in a summer

camp with the camp organizer A.

S: I want to sign her up for Spanish classes in the mornings, and rock climbing in the afternoons.

A: Okay, there are limited places in each activity based on age group, and some of the age groups have already filled up for rock climbing. How old is your daughter?

S: She's nine $\nearrow$
(24)

$$
\begin{aligned}
& \text { Confirmative rising declarative } \\
& \mathrm{S} \text { and A are on their way to a birthday } \\
& \text { party for the daughter of A's friend. } \\
& \text { They stop at a store to get a birthday } \\
& \text { card. As they are both scanning the } \\
& \text { display for a card for the correct age, } \\
& \mathrm{S} \text { is trying to remember how old the } \\
& \text { girl has just turned, and he thinks he } \\
& \text { remembers A telling him that she just } \\
& \text { turned nine, but he wants to confirm it. } \\
& \mathrm{S} \text { : She's nine } \nearrow
\end{aligned}
$$

It is clear to anyone who hears S's utterances in (22) and (23) that they are used by S to commit to the propositional content that she is nine, and thus make an assertion. Moreover, S's utterances in (24) and (25) are clearly used to ask questions about whether or not the proposition that she is nine is true. On top of this, both of the rising declaratives in (23) and (25) have some extra effects not necessarily present in (22) and (24), as discussed in (9) and (10) above: (23) raises a second issue. (25) conveys a bias that the propositional content of the declarative is true in the form of an expectation that A will commit to it.

My goal is to explain these discourse effects while maintaining stable denotations for declaratives, interrogatives, and the rise ' $\nearrow$ ', along with a single convention of use for declaratives and interrogatives. Given the identical form but very different discourse effects of (23) and (25), this goal cannot be achieved if illocutionary force is completely determined by the combination of clause type, intonation, and convention of use. My solution to this is to have the aforementioned components only bring us part of the way there. The audience of an utterance needs to rely on these components plus some amount of pragmatic inference to determine what a speaker intends to do with their utterance. 
By virtue of all being formed from declaratives, S's utterances in (22), (23), and (25) all denote the same truth-conditional content, \{that she is nine , while the polar interrogative in (24) denotes $\{$ that she is nine, that she is not nine $\}$. In each case, these semantic denotations are added to the top of the table by the utterance function in (20). Since the utterance function does not build in a notion of commitment, S's commitment to the proposition that she is nine in (22) and (23) will need to be achieved in another way. A key component here is the support requirement in (21), which applies pressure for an interlocutor to commit to a proposition in the topmost issue on the table, here, the proposition that she is nine.

In the specific case of (22), S uses falling intonation, which as the unmarked default, does not convey that $\mathrm{S}$ lacks commitment to a proposition $q$ that would help settle the QUD in $c$. Thus, in uttering the falling declarative, $\mathrm{S}$ has raised an issue that contains a proposition that would settle the QUD ("How old is your daughter?"), and S has not conveyed any lack of commitment. Given the support requirement, we infer that $\mathrm{S}$ did this because $\mathrm{S}$ intends to commit to the proposition that she is nine. The idea is that, given the goal of conversation to grow the common ground and the means by which this is done, and given that the speaker had the choice to use the rise ' $\nearrow$ ' to convey lack of commitment but didn't, we assume that utterances of falling declaratives that could advance the conversation by addressing the QUD are made because the speaker intends to make a commitment to the propositional content themselves. Thus the speaker commitment that usually follows from the utterance of a falling declarative is derived rather than built into a convention of use for declarative clauses. ${ }^{5}$

I will return to the assertive rising declarative in (23) after discussing the polar interrogative in (24) and the inquisitive rising declarative in (25). For (24), the support requirement applies pressure for an interlocutor to commit to either proposition in that she is nine, that she is not nine $\}$. The rise ' $\nearrow$ ' further conveys that $\mathrm{S}$ lacks commitment to a proposition $q$ that would help settle the QUD in $c$. Recall that the default assumption in (19-c) is that this lack of commitment is about the content of the utterance itself, which here would be the content proposition of the interrogative, that she is nine. There's no reason in (24) to think that the default assumption does not hold, so the rise signals that $S$ lacks commitment to this proposition. Furthermore, there's no reason to suspect that $S$ intends to commit to the negative answer that she is not nine. There are two reasons for this. One is that if they did intend to do so, they could have uttered a declarative with that content, raising only a singleton set issue which more easily leads to the inference that $S$ intends to make a commitment, as described for (22). The second reason is that world knowledge tells us that $\mathrm{S}$ is in an epistemically inferior position with respect to either proposition in that she is nine, that she is not nine $\}$ in the context of (24). Thus it is natural for $\mathrm{S}$ to raise an issue without intending to resolve it themselves (cf. Hudson (1975), Gunlogson (2008) who take this lack of epistemic authority to be constitutive of question asking). Given the pressure for someone to commit to either answer, we infer that $\mathrm{S}$ intends for $\mathrm{A}$ to make a commitment by providing an answer.

The inquisitive rising declarative in (25) functions similarly to the polar interrogative. The default assumption about the rise holds so that $S$ signals lack a commitment to the proposition that she is nine. Thus, $\mathrm{S}$ will not satisfy the support requirement by making a commitment, and

\footnotetext{
${ }^{5}$ In certain contexts, falling declaratives are used to ask questions by making dependent commitments (Gunlogson 2008). The existence of such uses further bolsters the view here that speaker commitment needs to be derived from rather than hardcoded into (falling) declaratives or their conventions of use.
} 
so given the pressure for someone to commit, we infer that $\mathrm{S}$ intends for A to make a commitment by providing an answer. The difference between (24) and (25) is that the latter is a singleton set, so the only choice that $\mathrm{S}$ gives to $\mathrm{A}$ for resolving the issue is to commit to the proposition that she is nine. Thus, the choice to use the rising declarative instead of the polar interrogative conveys S's expectation that A will commit to the proposition denoted by the declarative, the distinguishing contextual bias feature in (9). If $\mathrm{S}$ did not have this bias, they could have used the polar interrogative instead (cf. Westera $(2017,2018)$ and Rudin (2018) who give similar analyses of the contextual bias of rising declaratives).

As for the assertive rising declarative in (23), the support requirement applies pressure for some interlocutor to commit to the proposition that she is nine, and the rise signals that $\mathrm{S}$ lacks commitment to a proposition $q$ that would help settle the QUD in $c$. Unlike for (24) and (25), the default assumption that this lack of commitment is about the content of the declarative cannot be made here. The reason is that $\mathrm{S}$ knows much better than A how old S's own daughter is. Since S's lack of commitment is not about the content of the declarative, we infer that $S$ intends to commit to that content themselves, for the same reasons as for (22), namely $S$ raised a proposition that would answer the QUD that $S$ is in a position to commit to.

Finally, we need to resolve what the lack of commitment signaled by S's use of the rise in (23) is about. Recall that in (23), the goal is to get S's daughter enrolled in rock climbing (in QUD form, “Can I enroll my daughter in rock climbing?"). A has said that some age groups are already filled and asked a local question, the daughter's age. S's answer that she's nine doesn't settle the larger QUD. We infer the proposition that S lacks commitment to based on what it would have to be in order to combine with the proposition that she is nine to settle the QUD, as required by (19-b-i). The proposition that there is still room in the 9-year-old's rock climbing group fits this bill. Thus whether this proposition is true is the second issue raised by the assertive rising declarative, the final meaning generalization in (10).

Compare the above to another unified account depending on pragmatic derivation. For Westera $(2017,2018)$, the rise signals that the speaker is suspending or violating a Gricean maxim. Inquisitive RDs and polar interrogatives violate quality, while the assertive RD in (4) violates relevance, and that in (5) violates quantity. However, it is unclear how this account applies to (23) and (16), as S's utterances do not seem to violate any maxims. In particular, S's contribution is as informative as is required, and it clearly also makes a relevant contribution.

5. Conclusion. In this paper, I have argued that inquisitive and assertive rising declaratives cannot be distinguished on intonational grounds, and thus a unified account is preferable. Then I showed that giving a unified account based on stable meanings for declaratives, interrogatives, and rising intonation, as well as a single convention of use for declaratives and interrogatives, requires a dose of pragmatic flexibility: Illocutionary force cannot be determined by conventional content and utterance function alone. Audiences must infer the speaker's intended force based on both conventions and pragmatics. Due to space limitations, a fuller discussion of ramifications and of prior accounts is left to future work.

\section{References}

Boersma, Paul \& David Weenink. 2020. Praat: Doing phonetics by computer [computer program]. Version 6.1.16.

Farkas, Donka \& Kim Bruce. 2010. On reacting to assertions and polar questions. Journal of Semantics 27(1). 81-118. https://doi.org/10.1093/jos/ffp010. 
Farkas, Donka F. \& Floris Roelofsen. 2017. Division of labor in the interpretation of declara- tives and interrogatives. Journal of Semantics 34(2). 237-289. https://doi.org/10.1093/jos/ffw012.

Goodhue, Daniel, Lyana Harrison, Y. T. Clémentine Su \& Michael Wagner. 2016. Toward a bestiary of English intonational contours. North East Linguistic Society (NELS) 46. 311-320.

Goodhue, Daniel \& Michael Wagner. 2018. Intonation, yes and no. Glossa: A Journal of General Linguistics 3(1). 5. https://doi.org/10.5334/gjgl.210.

Gunlogson, Christine. 2003. True to form: Rising and falling declaratives and questions in English. London: Routledge.

Gunlogson, Christine. 2008. A question of commitment. Belgian Journal of Linguistics 22(1). 101136.

Hamblin, Charles L. 1973. Questions in Montague English. Foundations of language 10(1). 41-53. Hirschberg, Julia \& Gregory Ward. 1995. The interpretation of the high-rise question contour in English. Journal of Pragmatics 24. 407-412. https://doi.org/10.1016/0378-2166(94)00056-K.

Hudson, Richard A. 1975. The meaning of questions. Language 51(1). 1-31. https://doi.org/10.2307/413148.

Jeong, Sunwoo. 2018. Intonation and sentence type conventions: Two types of rising declaratives. Journal of Semantics 35. 305-356. https://doi.org/10.1093/semant/ffy001 .

Krifka, Manfred. 2017. Negated polarity questions as denegations of assertions. In Chung-min Lee, Ferenc Kiefer \& Manfred Krifka (eds.), Contrastiveness in information structure, alternatives and scalar implicatures, 359-398. Berlin: Springer.

Lauer, Sven. 2013. Towards a dynamic pragmatics. Stanford, CA: Stanford University dissertation.

Malamud, Sophia A. \& Tamina Stephenson. 2015. Three ways to avoid commitments: Declarative force modifiers in the conversational scoreboard. Journal of Semantics 32(2). 275-311. https://doi.org/10.1093/jos/ffu002.

Pierrehumbert, Janet. 1980. The phonology and phonetics of English intonation. Cambridge, MA: MIT dissertation.

Pierrehumbert, Janet \& Julia Hirschberg. 1990. The meaning of intonational contours in the interpretation of discourse. In Philip R. Cohen, Jerry L. Morgan \& Martha E. Pollack (eds.), Intentions in communication, 271-311. Cambridge, MA: MIT Press.

Portner, Paul. 2018. Mood. Oxford: Oxford University Press.

Roberts, Craige. 1996/2012. Information structure in discourse: Towards an integrated formal theory of pragmatics. Semantics and Pragmatics 5(6). 1-69. https://doi.org/10.3765/sp.5.6.

Rudin, Deniz. 2018. Rising above commitment. Santa Cruz: University of California dissertation.

Stalnaker, Robert. 1978. Assertion. In Robert Stalnzker (ed.), Context and content: Essays on intentionality in speech and thought, 78-95. Oxford: Oxford University Press.

Truckenbrodt, Hubert. 2006. On the semantic motivation of syntactic verb movement to $\mathrm{C}$ in German. Theoretical Linguistics 32(3). 257-306. https://doi.org/10.1515/TL.2006.018.

Truckenbrodt, Hubert. 2012. Semantics of intonation. In Claudia Maienborn, Klaus von Heusinger \& Paul Portner (eds.), Semantics: An international handbook of natural language meaning, vol. 3, 2039-2969. Berlin: de Gruyter.

Ward, Gregory \& Julia Hirschberg. 1985. Implicating uncertainty: The pragmatics of fall-rise intonation. Language 61(3). 747-776. https://doi.org/10.2307/414489.

Westera, Matthijs. 2017. Exhaustivity and intonation: A unified theory. Amsterdam: University of Amsterdam dissertation.

Westera, Matthijs. 2018. Rising declaratives of the quality-suspending kind. Glossa: A Journal of General Linguistics 3(1). 121. http://doi.org/10.5334/gjgl.415. 